\title{
Haptic Interfaces for Virtual Environments: Perceived Instability and Force Constancy in Haptic Sensing of Virtual Surfaces
}

\author{
Hong Z. Tan, Haptic Interface Research Laboratory \\ Purdue University
}

\begin{abstract}
Haptic interfaces are becoming more commonplace in virtual environment and teleoperation systems. There is a growing need to not only continue to improve hardware platforms and rendering algorithms, but evaluate human performance with haptic interfaces. This review summarizes two recent studies inspired by perception problems in using haptic interfaces to interact with virtual environments. The first study evaluated perceived quality of virtual haptic textures and discovered several types of perceived instability and their sources. We found that the buzzing type of perceived instability was most likely due to the mechanical resonance of the haptic interface hardware, and the aliveness type of perceived instability due to our inability to sense the slight movements of our hands in free space. The second study focused on the motor strategy employed during interaction with a virtual surface via a force-feedback haptic interface. We found that users tended to maintain a constant penetration force into a virtual surface when interacting with the surface. This can result in a reversal in perceived relative surface heights if the taller surface is rendered with a lower stiffness, thereby resulting in an erroneous perception of the virtual environment being rendered. For both studies, possible solutions to improving human perception of virtual and remote objects via hardware and/or software are discussed.
\end{abstract}

Résumé Les interfaces haptiques sont de plus en plus communes dans les systèmes de réalité virtuelle et de téléopération. Il y a un besoin croissant de continuer non seulement à améliorer les plateformes matérielles et les algorithmes de rendu, mais aussi d'évaluer la performance humaine avec des interfaces haptiques. Cet article résume deux études récentes inspirées des problèmes de perception signalés lors de l'utilisation d'interfaces haptiques pour interagir avec des environnements virtuels. Dans la première étude, nous avons évalué la qualité perçue des textures haptiques virtuelles et avons découvert plusieurs types d'instabilité perçue et leurs sources. Nous avons constaté que le type bourdonnant d'instabilité perçue était vraisemblablement dû à la résonance mécanique du matériel de l'interface haptique, et que le type vivant d'instabilité perçue était dû à notre incapacité de sentir les légers mouvements de nos mains dans un espace libre. La deuxième étude portait sur la stratégie motrice utilisée durant l'interaction avec une surface virtuelle par le truchement d'une interface haptique à retour de force. Nous avons découvert que les utilisateurs ont tendance à maintenir une force de pénétration constante dans une surface virtuelle lorsqu'ils sont en interaction avec la surface. Cela peut occasionner une inversion des hauteurs relatives perçues si la surface la plus élevée est rendue avec une rigidité moindre, entraînant de ce fait une perception erronée de l'environnement virtuel rendu. Nous discutons, pour les deux études, des solutions matérielles et/ou logicielles possibles pour améliorer la perception humaine des objets virtuels et distants.

To faithfully emulate the feel, as well as the sight and sound, of remote or virtual objects in teleoperation and virtual environments requires multimodal interfaces that match the human sensory and motor capabilities. Over the past two decades, much progress has been made in haptic technologies and in our understanding of the human somatosensory system, both of which are essential to the development of effective and intuitive haptic interfaces that bridge the real and remote/virtual worlds. The state-of-the-art hardware and software systems for haptic virtual environments strive to provide a realistic perceptual experience to enable a user to interact with virtual objects in a natural and intuitive manner. Typical hardware platforms include force-feedback devices such as the PHANToM (SensAble Technologies, Woburn, MA), Omega (Force Dimension, Switzerland), HapticmaSTER (MOOG FCS, the Netherlands), MagLev (Berkelman \& Hollis, 2000), and VISHARD10 (Technical University of Munich, Germany) that emulate the exploration and manipulation of objects through a single contact point. Realizing the need for distributed 
contact displays, new area-based devices have emerged that vary surface friction (Glassmire, 2006), cause lateral skin deformation (Wang \& Hayward, 2006), and form pin arrays with a density that matches that of the mechanoreceptors in the fingertip (Pawluk, Buskirk, Killebrew, Hsiao, \& Johnson, 1998; Summers \& Chanter, 2002). From a software point of view, fast haptic rendering algorithms are needed in order to compute force and/or torque resulting from contacting an object with a complex geometric representation, and to simultaneously control hundreds of pins in an array display, while running a sufficiently high haptic update rate.

Studies on human haptic perception have played an important role in reaching the full potential of the stateof-the-art hardware/software systems by informing design trade-offs. For example, a recent study developed a haptic data compression scheme over the internet using knowledge of force magnitude JNDs (just noticeable differences). The authors faced the conflicting demands of realistic haptic rendering through high transfer rate of haptic data (position, velocity, force, torque, etc.) over the Internet and limited Internet bandwidth. They then proposed a compression scheme for force-data delivery called the "deadband" principle that transmitted force data only when the new sample differed from the most-recently sent sample by more than the JND for the sent sample (Hinterseer \& Steinbach, 2006). User testing with remote exploration of a smooth spherical object showed that packet rates could be reduced by up to $90 \%$ for the transmission of force-magnitude data without significantly affecting the perceived quality of the object. Further psychophysical experiments were conducted to take into account force direction in haptic data compression (Pongrac, Hinterseer, Kammerl, Steinbach, \& Farber, 2006).

Haptic virtual environments provide an excellent venue for testing the characteristics of human haptic perception because of their ability to simulate a large range of objects that are sometimes difficult to fabricate in the real world. Sometimes, characteristics of the haptic interface hardware or the rendering/control software introduce perceptual artifacts into the sensing task. These artifacts not only decrease perceptual realism, but can also compromise the validity of the haptic virtual environment as a vehicle for psychophysical investigation or for information transmission. In this article, we summarize two recent psychophysical studies of haptic virtual environments that illuminate the sources of perceptual artifacts and suggest a combined engineering and psychology approach to solving the perceptual artifact problem. In Study 1, we quantitatively characterize the instability a human user often experiences while interacting with a virtual textured surface rendered with a force-feedback haptic interface (Choi \& Tan, 2004, 2005). In Study 2, we introduce the idea of force constancy that provides a model for manual exploration of virtual and/or remote surfaces (Choi, Walker, Tan, Crittenden, \& Reifenberger, 2005; Walker \& Tan, 2004). Together, these two studies enable us to understand the capabilities and limitations of forcefeedback devices and rendering algorithms, and provide general methods for doing so.

Perceived Instability of Virtual Haptic Texture

Haptic texture rendering is a growing research field that holds much promise for enriching the sensory attributes of objects in a virtual environment and for allowing precise and systematic control of textured surfaces for psychophysical studies. A common problem that has been reported anecdotally by many researchers is the instability perceived by a human user while interacting with a virtual textured surface rendered with a force-feedback haptic interface (e.g., Wall \& Harwin, 2000; Weisenberger, Krier, \& Rinker, 2000). We use the term perceived instability to refer to any unrealistic sensations that cannot be attributed to the physical properties of a virtual object under examination.

There are generally two major sources of perceived instability during haptic texture rendering: improper environment modelling and unstable control of the haptic interface. The former refers to the fact that the mathematical model used to calculate the forces resulting from interacting with a virtual object is usually an approximation to the underlying physics, thereby introducing "errors" that may be perceived and attributed to the virtual object by the human user. The stability of the haptic interface from a control theoretic point of view is also a necessary condition for realistic rendering. Unbounded behaviour of a controller, unmodelled dynamics of a haptic interface, quantization noise of encoders, energy instilling effects of a zero-order-hold digital-to-analogue converter, and asynchronous switch time can all lead to control instability (Gillespie \& Cutkosky, 1996). In addition, human perception and decision processes also play important parts in the perceived quality of virtual haptic textures, and will be the focus of the discussion here. By combining psychophysical experimentation and engineering measurement, we investigated the conditions under which perceived instability occurred and the underlying causes.

\section{Method}

A PHANTOM force-feedback haptic interface (Model 1.0A, Sensable Technologies, Woburn, MA) was used to render virtual textured surfaces. It was equipped with a stylus as an interaction tool and encoders for sensing 


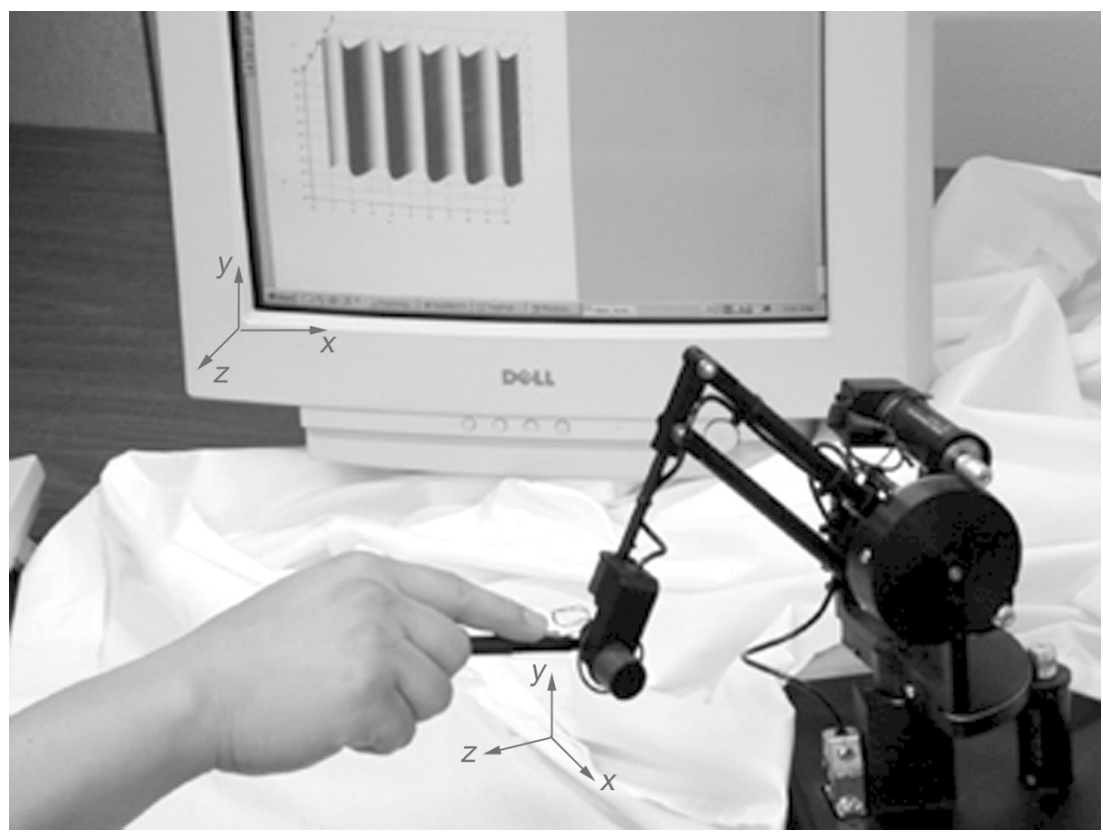

Figure 1. Illustration of experimental set-up. The user held a stylus attached to a PHANToM force-feedback device (foreground). The textured surface was a sinusoidal grating that varied only along the x-direction on a vertical plane (background, not shown during psychophysical experiments).

the position of the stylus tip (Figure 1). The virtual textures were modelled as a sinusoidal grating on a vertical surface facing the participant as shown on the computer monitor in the background of Figure 1. The grating heights along the $\mathrm{z}$-direction varied along the $\mathrm{x}$-axis but not the y-axis. The wavelength and amplitude of the gratings ranged between $1.0-4.0 \mathrm{~mm}$ and $0.5-2.0$ $\mathrm{mm}$, respectively. The force-feedback signals were calculated as follows. No force was exerted on the tip of the stylus as long as the stylus was outside the vertical textured surface (i.e., towards the participant's side). As the stylus moved inside the textured surface (i.e., along the $-z$ direction), a resistive force towards the participant (i.e., along the $+z$ direction) was exerted on the tip of the stylus. The force magnitude was determined by the product of the penetration depth along the $\mathrm{z}$ axis and the stiffness constant $(K$ in $\mathrm{N} / \mathrm{mm}$ ) of a linear elastic spring. This resistive force resulted in the user's perception of a virtual vertical surface that could not be easily penetrated. As the participant stroked the textured surfaces laterally (along the $\pm x$ directions), a vibratory signal determined by the surface amplitude and wavelength as well as the stroking velocity uniquely defined the roughness of the virtual texture.

The method of limits was used to find the maximum stiffness coefficients for different combinations of amplitude and wavelength values that resulted in stable virtual textures with no perceived instability. Three par- ticipants, one experienced with the PHANToM device and another two who had never used any haptic interface before, took part in the experiments. They could either stroke the textures from side to side (stroking mode, most frequently employed for texture perception and identification according to Lederman \& Klatzky, 1987) or explore the textures in any manner they wished (free exploration mode, more demanding on the perceived quality of the virtual textures because the participants' actions could not be easily predicted or incorporated into the rendering algorithm). They were instructed to regard any sensation that felt unrealistic based on their experience of real textures as an indication of perceived instability. Practice trials were provided in order for the participants to establish their response criteria. Qualitative descriptions of the types of perceived instability discovered were also collected.

Once the range of stiffness values for stable texture rendering was established, we investigated the attributes of the proximal stimuli experienced by the user's hand while exploring the virtual textured surface in an attempt to identify the sources of perceived instability. The PHANTOM device was instrumented with a triaxial force/torque sensor (Model nano17 with temperature compensation, ATI Industrial Automation, Apex, NC) and a triaxial accelerometer (Model 8794A500, Kistler, Blairsville, PA). Position, force and acceleration data from two experienced users of the PHANToM device 
— Power Spectrum ........ Human Detection Threshold

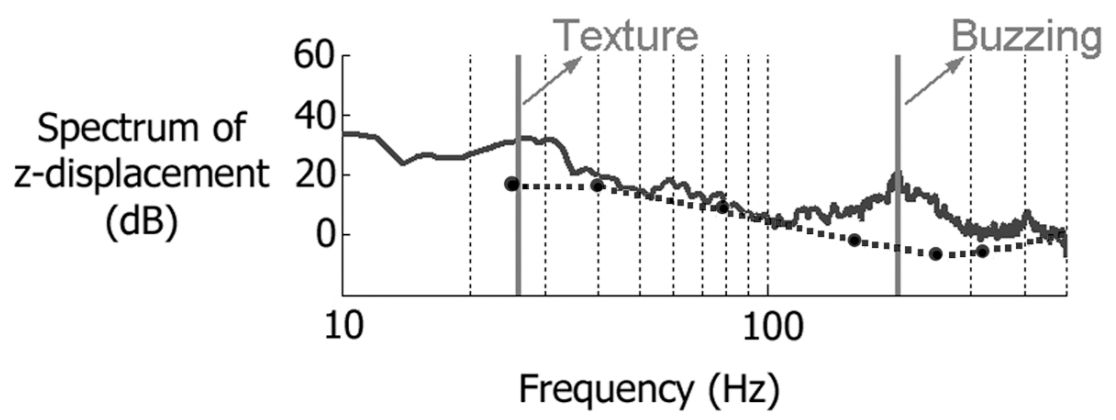

Figure 2. Power spectral density of z-position data. Shown are the spectral densities (solid line) and the detection thresholds at the thenar eminence (circles for measured thresholds with an interpolating dashed line). The two solid vertical lines mark the spectral components for texture perception and for perceived instability (buzzing), respectively. The remaining dashed vertical lines mark the frequencies at 20,30, 40, 50,60, 70, 80, 90, 100, 300, 400, and 500 Hz. (Replot of data in Choi \& Tan, 2004, Figure 9(c), upper panel.)

(one of whom participated in the psychophysical experiments) were measured and analyzed. The experienced users were preferred for the proximal stimuli measurements because they were required to explore the virtual textured surfaces in a well-controlled manner. Sometimes, they were required to move the stylus to a location where buzzing occurred and held the stylus there. At other times, the users were instructed to stroke the virtual textured surfaces back and forth with a constant velocity profile.

\section{Results}

The threshold values of $\mathrm{K}$, the maximum stiffness coefficient at which a stable rendering could be achieved, were quite small for all the conditions tested. They ranged from $0.0138 \mathrm{~N} / \mathrm{mm}$ to $0.4527 \mathrm{~N} / \mathrm{mm}$, resulting in textured surfaces that felt as soft as corduroy. In general, the threshold $\mathrm{K}$ values increased as texture amplitudes decreased and as texture wavelengths increased.

Participant debriefing at the end of the psychophysical experiments revealed several types of perceived instability, of which buzzing and aliveness are discussed here. Buzzing is characterized by a high-frequency noise that is felt in addition to the vibrations due to surface texture. Aliveness refers to a regular pulsating sensation coming from the textured surface as if a "live" artery runs underneath it.

The proximal stimuli for textures that contained buzzing noise exhibited prominent spectral peaks in two frequency regions. An example with data collected from a typical run is shown in Figure 2 where the spectral density function (solid line) for the z-position data and the human detection thresholds (dotted line interpolated between data points from Verrillo \& Gescheider, 1992) are plotted as a function of temporal frequency. There are two spectral peaks that are significantly above the human detection thresholds. The one around $26 \mathrm{~Hz}$ is due to the surface texture because its frequency is consistent with the ratio of average measured stroking velocity over texture wavelength. The one around $203 \mathrm{~Hz}$ is most likely the cause of the buzzing noise that is experienced as a high-frequency vibration. In general, we were able to isolate signal components in the frequency range of $192-240 \mathrm{~Hz}$ whenever the buzzing type of perceived instability was observed by the participants. This is the frequency range at which humans are most sensitive to vibrational stimulation due to activation of the Pacinian channel (Bolanowski, Gescheider, Verrillo, \& Checkosky, 1988). The spectral peak corresponding to texture information was found to be in the frequency range of $26-65 \mathrm{~Hz}$ at which stimulation is usually perceived to be "rough" and "fluttering" (Mountcastle, Talbot, Darian-Smith, \& Kornhuber, 1955). Since the frequency ranges for texture and buzzing perception are well separated along the frequency axis as well as in the neural mechanism mediating perception (fluttering and vibration, respectively), the participants were able to perceive the texture information in the presence of perceived instability and vice versa. It was hypothesized that the buzzing noise may be due to the instability of the PHANToM device itself. Further measurements of the frequency response of the PHANTOM indeed revealed a mechanical resonance at around $218 \mathrm{~Hz}$. Therefore, we can conclude that the buzzing type of perceived instability is 


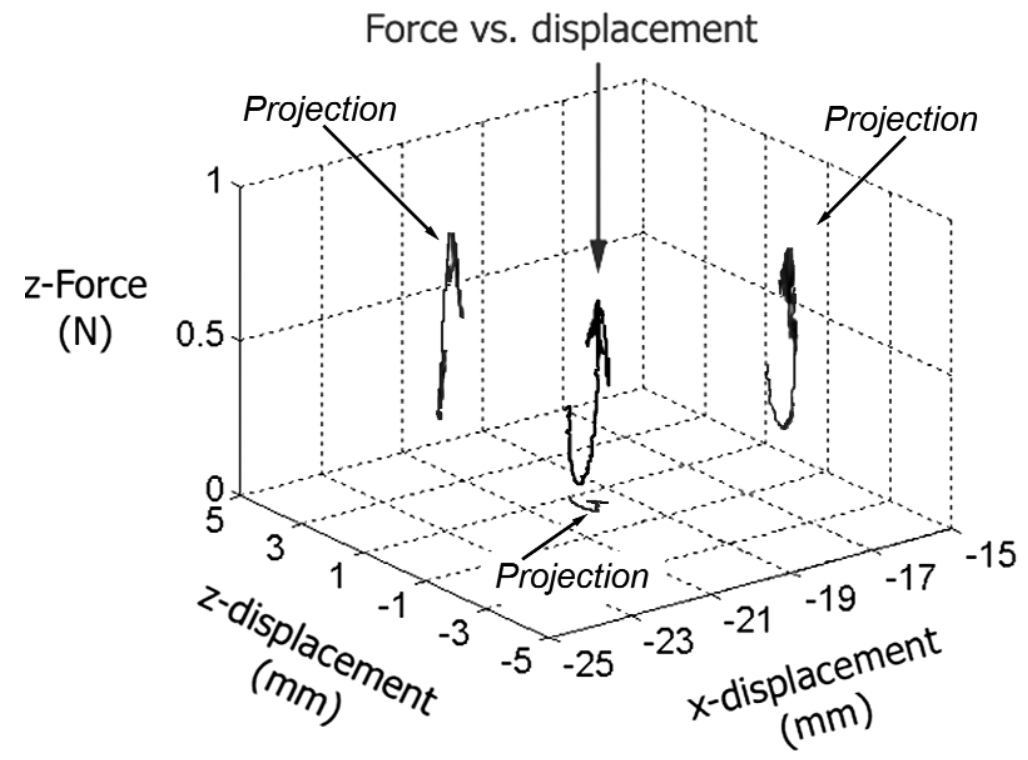

Figure 3. Characteristics of "aliveness" type of perceived instability. Data were collected when the participant held the PHANTOM stylus still inside a virtual textured plane. Shown are forces along the $\mathrm{z}$-direction as a function of the stylus tip position along the $\mathrm{x}$ and $\mathrm{z}$ directions (curve labelled "Force vs. displacement"). Also shown are the force versus $\mathrm{x}$ and force versus $\mathrm{z}$ projections as well as the displacement variations in the $\mathrm{x}-\mathrm{z}$ plane (curves labelled "Projection"). (Adapted from Choi \& Tan, 2005, Figure 8(a)).

most likely attributable to the instability of the haptic interface hardware.

The "aliveness" type of perceived instability often occurred when the stiffness coefficient of the rendering algorithm was slightly higher than the threshold value guaranteeing stable virtual textures. It is similar to the sensation experienced when one presses the index and middle fingers on the volar side of the wrist to feel the pulse: A pulsating force originating from under the skin surface can be felt by the fingers at a regular interval. This type of perceived instability was discovered when the participant pushed the stylus tip of the PHANToM inside the virtual textured surface and held the stylus still in space. The participant then experienced pulsating force variations that could not be attributed to the movement of the stylus, thereby getting an impression that the virtual textured surface was "alive." An example of the proximal stimuli associated with aliveness is shown in Figure 3 where the force variations are plotted as a function of the stylus tip positions (curve labelled "Force vs. displacement") along with the projections (curves labelled "Projection"). We did not plot force against the y-position since the texture model did not vary along the y-axis. It can be observed that a relatively large force variation occurred while the PHANTOM stylus barely moved at all. The change in force magnitude (up to $0.59 \mathrm{~N}$ ) was a result of the tremor of an unsupported hand in space (up to 0.56 and $0.94 \mathrm{~mm}$ in the $\mathrm{x}$ and $\mathrm{z}$ directions, respectively) while the participant held the stylus "stationary." While the force variation was large enough to be clearly perceived, the change in stylus tip position was not. The latter was supported by a recent study on human jointangle position resolution that derived a displacement JND of $2.2 \mathrm{~mm}$ at the fingertip of the index finger (Tan, Srinivasan, Reed, \& Durlach, in press). Therefore, the participant felt the "pulsing" of the virtual textured surface but was unaware of its source. Furthermore, the measured force and velocity data showed that "aliveness" could occur even when the PHANToM device and the rendering system were passive and stable (Choi \& Tan, 2005). We can now conclude that the aliveness type of perceived instability is a consequence of our inability to sense small movement caused by hand tremor. It is most likely due to the combination of our relatively high sensitivity to force variation and our relatively poor resolution of hand movement.

\section{Summary}

We investigated the problem of perceived instability during haptic texture rendering with the goals to quantify conditions under which virtual textures are per- 
ceived to be stable, to discover types of perceived instability, and to understand the sources of perceived instability. Our work was performed in the context of applying virtual textures to virtual object surfaces rendered with a force-feedback haptic interface, and of utilizing virtual textures as stimuli in psychophysical experiments on texture perception. Our measurements indicated that the buzzing type of perceived instability is quite intense at a relatively high frequency that coincided with one of the PHANToM device's structural resonance frequencies. The aliveness type of perceived instability, on the other hand, did not involve any discernable energy peak at a particular frequency. Instead, it was caused by our inability to perceive the hand tremor that resulted in the haptic rendering algorithm delivering perceivable force variations to the stylus. The presence of perceived instability seriously undermines the realism of virtual haptic textures and can lead to erroneous conclusions drawn from psychophysical experiments using such "contaminated" stimuli. By combining psychophysical and engineering approaches, we have taken the first step towards identifying, and pinpointing the sources of perceived instability associated with virtual objects.

Force Constancy in Exploring Virtual Surfaces

A unique aspect of haptics is that sensing and manipulation are integral components of the sense of touch. There is ample evidence that humans intuitively execute movement patterns that optimize the extraction of specific information about object properties. For example, studies of Exploratory Procedures (EPs) used in sensing objects and surfaces have shown that lateral stroking is best suited for judging surface roughness, compressing an object between fingers for judging its stiffness, etc. (Lederman \& Klatzky, 1987). While many of the EPs can be directly extended to teleoperated and virtual environments, what might be some of the additional principles that govern the way humans interact with virtual objects? The goal of this study was therefore to investigate how the constraints imposed by a haptic interface affect surface exploration procedures.

We observe that the way people interact with a virtual surface is fundamentally different from that with a real surface when the surfaces are reasonably hard to the touch. For ease of comparison, let us assume that both the real and virtual surfaces are explored with a point-contact stylus. No resistive force is felt through the stylus until it collides with an object. During exploration of a real surface such as a tabletop, the stylus tip remains in contact with the surface but does not penetrates it. The downward force exerted by the hand on the stylus is balanced by the upward reactive force exerted by the stiff tabletop on the same stylus. The stiffness of a force-feedback device, however, is limited by its mechanical structure as well as other factors such as force update rate and control algorithm. Consequently, when a user pushes a stylus into a virtual surface, the surface yields (e.g., like an elastic spring) until the restoring force exerted by the virtual surface on the stylus is large enough to resist the user's force. It follows that the stylus tip always penetrates a virtual surface before a feedback force can be generated to indicate the existence of the surface. Therefore, it is much more challenging to render a hard surface than a soft deformable surface. In both the real and virtual environments, the user forms a mental image of the surface topography based on the perceived trajectory of the stylus tip. It is important that the trajectory of the stylus be parallel to that of the virtual surface in order for the user to perceive the surface topography correctly, albeit at an offset in space. When the trajectory of the stylus tip ceases to be parallel to the virtual surface, the user's perception of the surface topography is distorted.

How can the stylus tip remain parallel to the virtual surface? We hypothesized that humans do so by maintaining a constant penetration force while exploring virtual surfaces. As long as surface topography is rendered with a penalty-based method (i.e., resistive force is calculated by multiplying penetration depth by a stiffness constant) and the stiffness is uniform across the entire surface, our force-constancy hypothesis predicts that the stylus tip will remain a constant distance below the actual surface topography. In other words, the trajectory of the stylus tip follows the surface topography faithfully. When the stiffness varies across the surface, however, our force-constancy hypothesis predicts distortion. This phenomenon was observed in a real-world data perceptualization (i.e., visualization with audio and haptic feedback) system. Specifically, we found that a more compliant surface that was slightly taller than an adjacent one was perceived to be shallower than the adjacent surface. Investigation of this distorted perception led to the formulation of our force-constancy hypothesis, which was validated in two experiments.

\section{Method}

A PHANToM force-feedback device (Desktop model, SensAble Technologies) was used for rendering virtual surfaces. The haptic stimuli consisted of two vertically adjoined planes facing the user, with either the left or the right plane being closer to the user. To eliminate a sudden change in rendered force across the boundary of the two planes, a small boundary region $(4 \mathrm{~mm}$ wide) was created to interpolate the two planes smoothly. In the first experiment, 10 participants were 


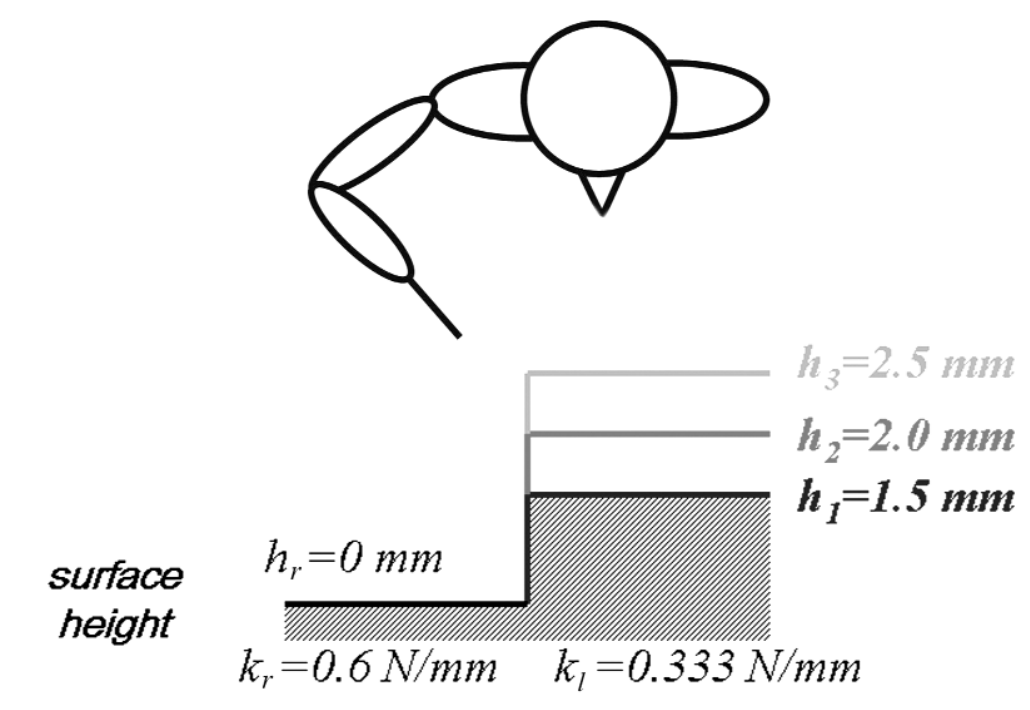

a)

b)

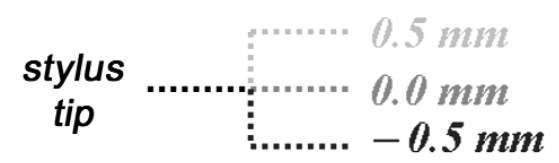

Figure 4. Illustration of stimuli used in the second experiment. (a) Bird's-eye view of the participant holding a stylus and the three cases of surface height. The right (from the participant's point of view) plane had a constant surface height and stiffness $\left(b_{r}\right.$ and $k_{r}$, respectively). The left plane had a different constant stiffness $\left(k_{l}\right)$. The left plane was $1.5,2.0$, or $2.5 \mathrm{~mm}$ (Conditions 1 to 3 , respectively) closer to the participant than the right plane. (b) Predicted probe-tip trajectory and hence perceived relative height of the two surfaces. According to our force constancy hypothesis (with a constant penetration force of $1.5 \mathrm{~N}$ ), the left plane would be perceived to be further away from the participant, at the same height, and closer to the participant, as compared to the right plane, under the three conditions shown in (a), respectively.

asked to stroke the two planes from left to right once, and the penetration depths were recorded at $1 \mathrm{kHz}$. The planes were always of equal distance from the user (i.e., the surface height difference remained 0 $\mathrm{mm}$ ), but the stiffness values of the left and right planes were randomly chosen from the following pairs with a constant difference of $0.2 \mathrm{~N} / \mathrm{mm}:(0.1,0.3),(0.2$, $0.4),(0.5,0.7),(0.6,0.8)$, and $(0.9,1.1) \mathrm{N} / \mathrm{mm}$. For each stiffness pair, the left plane was stiffer on about half of the trials, and more compliant on the remaining trials. The participants were asked to concentrate on the relative height of the two planes while stroking the vertical surface from left to right. Although the data on surface height judgment were not analyzed, the task nevertheless helped participants to optimize their movements for the perception of surface topography.

In the second experiment, we explicitly tested the distortions predicted by our force-constancy hypothesis. Because of the need to maintain a constant penetration force, three experienced users of the haptic device participated in this experiment. The participants were asked to stroke the two vertical planes once from left to right while maintaining a constant penetration force of $1.5 \mathrm{~N}$ via visual feedback. Their task was to discriminate the relative position of the two planes and report which plane (left or right) was perceived to be closer to their body. Three experimental conditions were tested, as illustrated in Figure 4(a). A total of 300 trials were collected per participant and per experimental condition. For each condition, the left plane was 


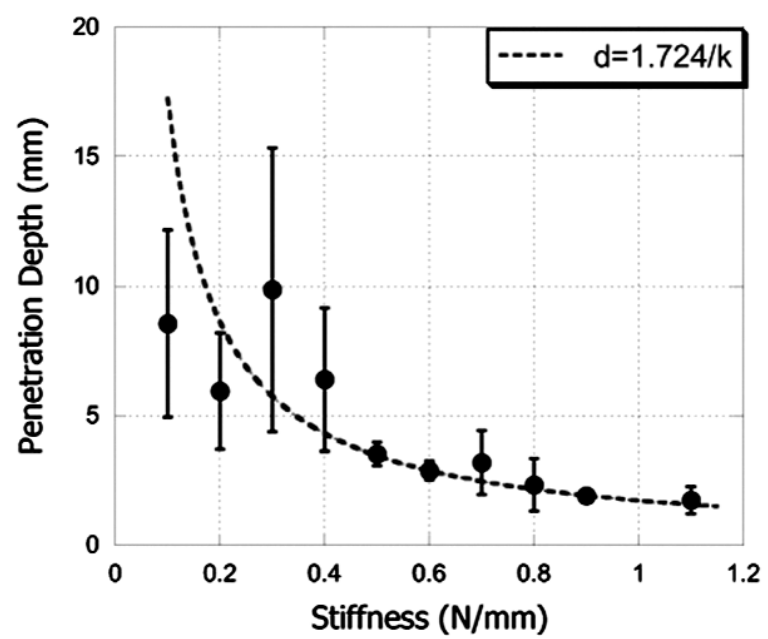

Figure 5. Results of the first experiment on our force-constancy hypothesis for one participant. Shown are the average penetration depths as a function of surface stiffness, along with standard deviations. The dashed curve corresponds to the best-fitting curve with an estimated penetration force of 1.724 N. (From Choi et al., 2005, Figure 7.)

always more compliant and closer to the user than the right one. The three conditions differed in the position of the left plane. Shown in Figure 4(b) are the predictions of the perceived relative height of the two planes in terms of the stylus tip trajectories. For example, in Condition 2, the stylus tip would penetrate the left plane by $1.5(\mathrm{~N}) / 0.333(\mathrm{~N} / \mathrm{mm})=4.5 \mathrm{~mm}$ and the right plane by $1.5(\mathrm{~N}) / 0.6(\mathrm{~N} / \mathrm{mm})=2.5 \mathrm{~mm}$. Since the left plane was taller than the right one by $h_{2}=2.0 \mathrm{~mm}$ to begin with, it was predicted that the stylus tip would travel a straight line, as shown in Figure 4(b). It follows that the participants would not be able to distinguish the height difference between the left and right planes in Condition 2.

\section{Results}

Figure 5 shows the results from the first experiment for one of the participants in the form of penetration depth $(d)$ versus surface stiffness $(k)$. In general, penetration depth decreased as surface stiffness increased, except for the two data points at the lowest stiffness values of 0.1 and $0.2 \mathrm{~N} / \mathrm{mm}$. Surfaces with these stiffness values were too soft to be well defined (e.g., like cotton balls), so it was not surprising that the penetration depth did not follow the general trend. The data could be well fitted by a curve assuming constant penetration force in the form of $d \cdot k=$ constant force. Data from other participants followed the same trend, and it was clear that every participant had a "preferred" constant penetration force that ranged between $0.87-2.23 \mathrm{~N}$

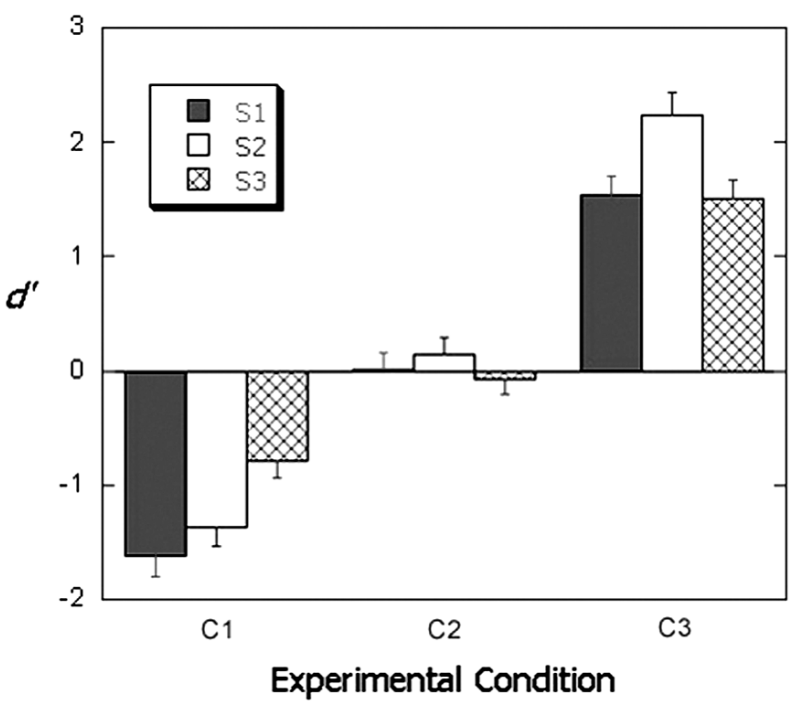

Figure 6. Average results of the second experiment on our forceconstancy hypothesis. Shown are the $\mathrm{d}^{\prime}$ scores for the three participants (S1, S2, and S3) under the three experimental conditions (C1, C2, and C3). (From Choi et al., 2005, Figure 8.)

for the 10 participants tested. Overall, the results from the first experiment confirmed that participants maintained a constant penetration force while exploring surfaces with different stiffness values.

The results from the second experiments are shown in Figure 6. The values of the sensitivity index $d^{\prime}$ for each participant and each experimental condition are shown with standard deviations. The general trend of the data was exactly as predicted by Figure 4(b). In Condition 1 where the left plane was predicted to be perceived as being further away from the participant as the right plane (i.e., a reversal in relative surface height), the average $d^{\prime}$ was -1.27 , indicating that the participants were able to detect the difference in surface height but misinterpreted the relative depth of the two planes. In Condition 2 where the stylus tip was predicted to traverse a straight line, the average $d^{\prime}$ was 0.03 , indicating that the participants could hardly discern the height difference between the two planes. In Condition 3 where no reversal of surface height was predicted, the average $\mathrm{d}^{\prime}$ was 1.76 , indicating that participants were able to sense and correctly interpret the relative height difference of the two planes. These results demonstrated that a more compliant but taller surface may be perceived as taller, at the same height, or shallower than an adjacent surface depending on the relative height difference between the two surfaces, due to the fact that the stylus tip would penetrate a more compliant surface deeper than it would a stiffer surface. For all three conditions tested, our force-constancy hypothesis was able 
to predict correctly when the reversal in perceived surface height would occur, once the penetration force was known (or controlled to be at a certain level as was the case during this experiment).

\section{Summary}

Our force-constancy hypothesis states that the user of a force-feedback device maintains a constant penetration force when stroking virtual surfaces in order to perceive their topography. This hypothesis can be used to explain the phenomenon that when two adjacent surfaces differ in both height and stiffness, the taller but more compliant surface could be perceived to be shallower, at the same height, or taller than the other surface, depending on how much taller it is than the other surface. The results from the two experiments presented here were consistent with the predictions of our force-constancy hypothesis. Our findings underscore the importance of understanding the interplay of haptic rendering parameters as we venture into the scientific perceptualization of more complex data sets.

One caveat of our force-constancy hypothesis is that the constant penetration force has to be known before the penetration depths can be calculated and predictions be made on whether a reversal in perceived surface height may occur. This was dealt with in the second experiment by requiring the participants to maintain a known constant penetration force using visual feedback signals. In a real application, it may be necessary to estimate the preferred constant penetration force by asking a user to stroke a virtual terrain for a short period of time. Moreover, we have observed that inexperienced users tended to exhibit a more variable penetration force initially, but converged to a more consistent force level as they became familiar with the force-feedback device. For these users, it will likely take longer for a computer program to establish their preferred penetration force level.

\section{Concluding Remarks}

In this review, we have provided a description of two recent studies that investigated human performance with haptic interfaces for virtual environment and teleoperation. Our studies were motivated by the real-world problem of perceived instability (Study 1) and distorted perception of relative surface height (Study 2). In each case, we have hypothesized the sources of the problems, and used a combined psychophysical and engineering approach to characterize the proximal stimuli responsible for observed problems in perception. In Study 1, additional sensors were placed near the stylus of a force-feedback device to record force and acceleration, in addition to using the existing encoders on the haptic interface for recording position and velocity data, in order to understand the signals experienced by the mechanoreceptors in the hand. We have found it insightful to analyze these signals in the spectral domain so the signal intensities can be considered relative to the human detection thresholds and the type of mechanoreceptors mediating perception at the corresponding frequencies. Once the sources of perceived instability were found, engineering solutions can be devised. For example, given that the buzzing type of perceived instability usually occurs at a frequency that is much higher than that of the texture signal, it may be advantageous to lowpass-filter force signals in the control loop. The finding that the buzzing noise may have originated from the mechanical resonance of the haptic interface itself advocates for new devices with resonant frequencies beyond several hundred hertz. The aliveness type of perceived instability requires a more software-based solution. Specifically, the haptic rendering loop can stop generating new force values when the displacement of the stylus tip is within a millimeter or two, because the user may very well be holding the stylus stationary during that time period and hence should not experience any force variations.

The investigation of proximal stimuli in Study 2 focused on the trajectory of the stylus tip as the source of information for perception, as opposed to the mathematical definition of virtual surfaces in the haptic rendering algorithm. As more and more parameters are introduced in rendering haptic virtual environment, it is a useful exercise to simulate the signals coming from the stylus tip to discover possible interactions among the rendering parameters. In the case considered here where only two parameters, namely the surface topography and stiffness, are varied, the surface height map can be "prewarped" in order to counteract the variation in penetration force due to nonuniform surface stiffness distribution based on our force-constancy hypothesis. This assumes, as discussed above, that the penetration force can be found by asking a user to interact with a virtual surface initially. A recent study has shown that such a compensation strategy works (Cheon \& Choi, 2006). Finally, it is important to note that the compensation algorithm can prewarp the surface topography but not stiffness distribution. This is because the surface stiffness is usually perceived by tapping the stylus against the surface; therefore any change in the stiffness map will result in distorted perception of stiffness distribution. Prewarping surface topography works because a user does not have direct access to the mathematical definition of a surface height map and can only perceive the trajectory of the stylus tip. The validity of perception can be assured as long as the stylus tip follows the intended shape of the surface topography.

The sense of touch conveys a wealth of informa- 
tion about the environment that we live in. Force-feedback devices can only emulate a small portion of that experience. As haptic researchers continue to develop more varieties of haptic interfaces, improve on haptic control and rendering algorithms, and design haptic interaction paradigms, a combined psychophysical and engineering approach will continue to play an important role in validating perception of virtual environments and in devising solutions to perceptual artifacts originating from hardware and software that do not perfectly match the human sensory-motor capabilities.

The author wishes to thank an anonymous reviewer and Dr. Roberta Klatzky for their careful reading of an earlier manuscript and excellent suggestions. The studies reported here were partly supported by a National Science Foundation (NSF) Faculty Early Career Development (CAREER) Award under Grant No. 9984991-IIS, an NSF award under Grant No. 0098443-IIS, a NASA award under Grant No. NCC 2-1363, the Birck Nanotechnology Center at Purdue University, and the Envision Center for Data Perceptualization at Purdue University. Correspondence regarding this article should be addressed to Dr. Hong Z. Tan, Electrical Engineering Building, 465 Northwestern Avenue, Purdue University, West Lafayette, IN 47907-2035 (E-mail: hongtan@purdue.edu).

\section{References}

Berkelman, P. J., \& Hollis, R. L. (2000). Lorentz magnetic levitation for haptic interaction: Device design, performance, and integration with physical simulations. International Journal of Robotics Research, 19, 644-667.

Bolanowski, S. J., Jr., Gescheider, G. A., Verrillo, R. T., \& Checkosky, C. M. (1988). Four channels mediate the mechanical aspects of touch. Journal of the Acoustical Society of America, 84, 1680-1694.

Cheon, J., \& Choi, S. (2006). Perceptualizing a "haptic edge" with varying stiffness based on force constancy. In Z. Pan, A. D. Cheok, M. Haller, R. W. H. Lau, H. Saito \& R. Liang (Eds.), Advances in Artificial Reality and TeleExistence, Proceedings of the 16th International Conference on Artificial Reality and Telexistence (ICAT 2006) (Vol. 4282). Hangzhou, China, November 29 December 1, 2006. Berlin: Springer.

Choi, S., \& Tan, H. Z. (2004). Perceived instability of virtual haptic texture. I. Experimental studies. Presence: Teleoperators and Virtual Environments, 13, 395-415.

Choi, S., \& Tan, H. Z. (2005). Perceived instability of virtual haptic texture. II. Effect of collision detection algorithm. Presence: Teleoperators and Virtual Environments, 14, 463-481.

Choi, S., Walker, L. A., Tan, H. Z., Crittenden, S., \& Reifenberger, R. (2005). Force constancy and its role on haptic perception of virtual surfaces. ACM Transactions on Applied Perception, 2, 89-105.

Gillespie, R. B., \& Cutkosky, M. R. (1996). Stable user-specific haptic rendering of the virtual wall. Proceedings of the ASME International Mechanical Engineering Congress and Exhibition (Vol. 58, pp. 397-406). American Society of Mechanical Engineerings.

Glassmire, J. (2006). Study and design of a variable friction haptic display. Unpublished master's thesis, Northwestern University, Evanston, IL.

Hinterseer, P., \& Steinbach, E. (2006). A psychophysically motivated compresssion approach for 3D haptic data. Proceedings of the International Symposium on Haptic Interfaces for Virtual Environment and Teleoperator Systems (pp. 35-41). New York: IEEE Computer Society.

Lederman, S. J., \& Klatzky, R. L. (1987). Hand movements: A window into haptic object recognition. Cognitive Psychology, 19, 342-368.

Mountcastle, V. B., Talbot, W. H., Darian-Smith, I., \& Kornhuber, H. H. (1955). Neural basis of the sense of flutter-vibration. Science, 155, 597-600.

Pawluk, D. T. V., Buskirk, C. P. V., Killebrew, J. H., Hsiao, S. S., \& Johnson, K. O. (1998). Control and pattern specification for a high density tactile array. In R. J. Furness (Ed.), Proceedings of the ASME dynamic systems and control division (Vol. 64, pp. 97-102). Anaheim, CA: ASME.

Pongrac, H., Hinterseer, P., Kammerl, J., Steinbach, E., \& Farber, B. (2006). Limitations of human 3D-force discrimination. Proceedings of the Second International Workshop on Human-Centered Robotic Systems. Munich, Germany: Technical University of Munich.

Summers, I. R., \& Chanter, C. M. (2002). A broadband tactile array on the fingertip. Journal of the Acoustical Society of America, 112, 2118-2126.

Tan, H. Z., Srinivasan, M. A., Reed, C. M., \& Durlach, N. I. (in press). Discrimination and identification of finger joint-angle positions using active motion. ACM Transactions on Applied Perception.

Verrillo, R. T., \& Gescheider, G. A. (1992). Perception via the sense of touch. In I. R. Summers (Ed.), Tactile aids for the bearing impaired (pp. 1-36). London: Whurr Publishers.

Walker, L., \& Tan, H. Z. (2004). A perceptual study on haptic rendering of surface topography when both surface height and stiffness vary. Proceedings of the 12th International Symposium on Haptic Interfaces for Virtual Environment and Teleoperator Systems (HAPTICS '04) (pp. 138-145). IEEE Computer Society.

Wall, S. A., \& Harwin, W. S. (2000). Effects of physical bandwidth on perception of virtual gratings. In S. S. Nair (Ed.), Proceedings of the Ninth (9th) International Symposium on Haptic Interfaces for Virtual Environment and Teleoperator Systems, American Society of Mechanical Engineers Dynamic Systems and Control 
Division (Vol. 69-2, pp. 1033-1039). Orlando, FL: ASME. Wang, Q., \& Hayward, V. (2006). Compact, portable, modular, high-performance, distributed tactile transducer device based on lateral skin deformation, Proceedings of the International Symposium on Haptic Interfaces for Virtual Environment and Teleoperator Systems (pp. 6772). New York: IEEE Computer Society.
Weisenberger, J. M., Krier, M. J., \& Rinker, M. A. (2000). Judging the orientation of sinusoidal and square-wave virtual gratings presented via 2-DOF and 3-DOF haptic interfaces. Haptics-e, 1(4). Available at: http://www.haptics-e.org. 